\title{
Efficacy of intraoperative dexmedetomidine infusion on visualization of the surgical field in endoscopic sinus surgery
}

\author{
Hyunzu Kim ${ }^{1}$, Sang-Hee $\mathrm{Ha}^{2}$, Chang-Hoon Kim³ ${ }^{3}$ Sang-Hoon Lee ${ }^{1}$, and \\ Seung-Ho Choi ${ }^{2}$ \\ Department of Anesthesiology and Pain Medicine, ${ }^{1}$ Kangdong Sacred Heart Hospital, Hallym University College of \\ Medicine, ${ }^{2}$ Yonsei University College of Medicine, ${ }^{3}$ Department of Otorhinolaryngology, Medical Research Center, \\ Yonsei University College of Medicine, Seoul, Korea
}

Background: In endoscopic sinus surgery, visualization of the surgical field is a major concern, as surgical bleeding is the cause of many complications. The purpose of this study was to compare the effects of dexmedetomidine and remifentanil on the visualization of the surgical field in endoscopic sinus surgery.

Methods: Forty-three patients were prospectively enrolled and randomly allocated to the dexmedetomidine or remifentanil group and general anesthesia was induced and maintained using a propofol target-controlled infusion. In the dexmedetomidine group, dexmedetomidine was loaded for $5 \mathrm{~min}$ and a continuous infusion was administered. In the remifentanil group, a remifentanil target-controlled infusion was used. After completion of the operation, the satisfaction with the visualization of the surgical field was assessed on a numeric rating scale, from 0 (= worst) to 10 (= best). The mean blood pressure, heart rate, recovery profiles, and postoperative pain score were recorded.

Results: Satisfaction score for visualization by numeric rating scale was not significantly different between the two groups $(\mathrm{P}=0.95)$. There were no differences in the mean blood pressure and heart rate. The extubation time was significantly shorter in the dexmedetomidine group $(8.4 \pm 1.8 \mathrm{~min})$ than in the remifentanil group $(11.9 \pm 5.4 \mathrm{~min})(\mathrm{P}=0.04)$. Except for the extubation time, the recovery profiles of the two groups were comparable.

Conclusions: Continuous infusions of dexmedetomidine provide a similar visualization of the surgical field and hemodynamic stability as remifentanil target-controlled infusions in patients undergoing endoscopic sinus surgery.

Key Words: Blood loss, Dexmedetomidine, Remifentanil, Sinusitis.

Corresponding author: Seung-Ho Choi, M.D., Ph.D.

Department of Anesthesiology and Pain Medicine, Yonsei University College of Medicine, 50-1, Yonsei-ro, Seodaemun-gu, Seoul 03722, Korea

Tel: 82-2-2228-2420, Fax: 82-2-2227-7897

E-mail: csho99@yuhs.ac

Received: April 8, 2015.

Revised: 1st, May 26, 2015; 2nd, June 29, 2015; 3rd, July 3, 2015.

Accepted: July 5, 2015.

Korean J Anesthesiol 2015 October 68(5): 449-454

http://dx.doi.org/10.4097/kjae.2015.68.5.449

\section{Introduction}

Endoscopic sinus surgery (ESS) has become the most common technique for the surgical management of chronic rhinosinusitis [1]. Visualization of the surgical field is a major concern during ESS, as surgical bleeding is an important cause of various complications, including vessel or nerve damages and cerebrospinal fluid leakage [2]. Moreover, improved visualization of the surgical field may bring about not only fewer complications, but also a reduced surgical time, faster postoperative recovery, and a reduced relapse rate $[3,4]$.

(c) This is an open-access article distributed under the terms of the Creative Commons Attribution Non-Commercial License (http://creativecommons.org/ licenses/by-nc/4.0/), which permits unrestricted non-commercial use, distribution, and reproduction in any medium, provided the original work is properly cited. 
The nasal cavity is a limited space normally available through a nostril, and the surgeon can only use one hand to perform the work, as the other one holds the scope. The techniques used to control bleeding in other parts of the body cannot be used for surgeries of the nasal cavities and paranasal sinuses [5]. Many studies have ascertained the efficacy of specialized methods for reducing surgical bleeding during ESS. Above all, Guven et al. [6] found a lower heart rate (HR) and mean blood pressure (MBP) in patients receiving dexmedetomidine, and suggested that the administration of the drug may cause less bleeding.

The purpose of this prospective randomized controlled study was to compare the effects of intravenous dexmedetomidine as an adjuvant agent for propofol anesthesia and propofol-remifentanil TCI on the visualization of the surgical field, hemodynamic changes, and recovery profiles in the recovery period from general anesthesia in patients undergoing ESS.

\section{Materials and Methods}

This study was approved by the University Hospital Ethic Committee and was registered at ClinicalTrials.gov. Written informed consent was obtained from all patients. Forty-three patients aged 20-70 years old of American Society of Anesthesiologists physical status I and II who had been scheduled to undergo endoscopic sinus surgery, were prospectively enrolled in this study. The patients with a body mass index of 30 or higher, with congestive heart failure, a bradycardia rate under 50 per minute, first degree or more atrioventricular block, uncontrolled high blood pressure, clotting disorders, allergic fungal sinusitis or infection with multiple nasal polyps, and those who did not understand the meaning of the study or could not read the agreement, were excluded from the study. The patients' preoperative computed tomography scores (Lund-Mackay Computed Tomography Staging System) were evaluated and recorded [7].

None of the patients were premedicated upon arrival in the operating room. The routine anesthesia monitoring consisted of an electrocardiogram, pulse oximetry $\left(\mathrm{SpO}_{2}\right)$, noninvasive arterial pressure, partial pressure of end-tidal $\mathrm{CO}_{2}\left(\mathrm{EtCO}_{2}\right)$ and neuromuscular monitoring by train-of-four (TOF) (TOF-Watch, Organon Ltd, Doublin, Ireland). The anesthetic depth was monitored on the Bispectral Index (BIS VISTA Monitoring System, Aspect Medical Systems Inc, Norwood, MA, USA). A commercial TCI system (Orchestra Base Primea, Fresenius Vial, Brezins, France) was used to maintain the effect-site concentration (Ce) of propofol according to Schnider's pharmacokinetic model and that of remifentanil according to Minto's model.

Using a computer-generated randomization table, the patients were randomly allocated to one of two groups: dexmedetomidine or remifentanil. The anesthesia was induced and maintained using a propofol TCI to maintain a BIS range of 45 to 55. In the dexmedetomidine group, a bolus of fentanyl $1 \mu \mathrm{g} /$ $\mathrm{kg}$ and a loading of dexmedetomidine $0.5 \mu \mathrm{g} / \mathrm{kg}$ of loading for $5 \mathrm{~min}$ and a continuous infusion of $0.5 \mu \mathrm{g} / \mathrm{kg} / \mathrm{hr}$ administered. Labetalol $5 \mathrm{mg}$ was administered as needed to maintain the HR and MBP within $20 \%$ of the preoperative resting values. In the remifentanil group, a Ce of remifentanil TCI was controlled to achieve a HR and MBP within 20\% of the preoperative baseline values. After rocuronium $0.6 \mathrm{mg} / \mathrm{kg}$ was administered intravenously, the patients were intubated using an endotracheal tube with an internal diameter of $7.5 \mathrm{~mm}$ (for males) or $6.5 \mathrm{~mm}$ (for females). A cuff was inflated to maintain the pressure between 20-25 $\mathrm{cmH}_{2} \mathrm{O}$ with a manometer ( $\mathrm{Hi}-\mathrm{Lo}^{\mathrm{TM}}$ Hand Pressure Gauge, VBM Medizintechnik GmbH, Sulz a.N., Baden-Württemberg, Germany). Mechanical ventilation was maintained at a tidal volume of $8 \mathrm{ml} / \mathrm{kg}$ and $\mathrm{EtCO}_{2}$ of $35-45 \mathrm{mmHg}$, with an air/ oxygen mixture $\left(\mathrm{FIO}_{2}: 0.5\right)$.

The endoscopic surgeries were performed by a single surgeon following a standardized method, and the surgeon used soaking sponges with a $1: 100,000$ epinephrine solution as a vasoconstrictor.

Five minutes before the end of the surgery, the propofol was titrated to achieve the target BIS of 60 . In the dexmedetomidine group, the dexmedetomidine infusion was stopped. In the remifentanil group, the Ce of remifentanil was titrated to $2.0 \mathrm{ng} / \mathrm{ml}$ and was maintained until extubation. In both groups, after all surgical procedures were completed, the propofol was discontinued and the fresh gas flow was adjusted to $10 \mathrm{~L} / \mathrm{min}$. Thereafter, another anesthesiologist who was blinded to the patient groups performed the remaining anesthetic recovery processes. The anesthesiologist was blinded to the medications related to the study, as the syringe pumps were placed in a box and were invisible from the outside. The researcher in charge handled the medications and assisted the anesthesiologist. After confirming neuromuscular function by using the TOF response test, respiration was assisted by manual ventilation. If the patients opened their eyes in response to verbal requests and if their respiration was adequate, the trachea was extubated following cuff deflation. The patients in both groups were monitored while receiving $100 \%$ oxygen for $5 \mathrm{~min}$, and were transferred to the postanesthetic care unit (PACU). If the patients asked for rescue analgesics and their postoperative pain score was above 5 points on the visual analogue scale (VAS), fentanyl $1 \mu \mathrm{g} / \mathrm{kg}$ was administered.

After completion of the operation, the satisfaction with the visualization of the surgical field was assessed by the surgeon on a numeric rating scale (NRS) from 0 (= worst) to 10 (= best). The HR and MBP were recorded at the following times: T0; before induction, T1; before intubation, T2; after intubation, T3; after local injection, T4; 5 min after local injection, T5; 10 min after local injection, T6; $20 \mathrm{~min}$ after local injection, T7; 5 min 
before end of operation, T8; end of operation, T9; extubation, T10; admission to the PACU. The recovery profiles, including the time to awakening (from discontinuation of propofol to eye opening in response to verbal command), the time to extubation (from discontinuation of propofol to extubation), the coughing reflex at extubation, and the respiration rate and sedation scale [8] in the PACU, were measured and recorded. The consumption of anesthetic agents such as propofol, remifentanil, dexmedetomidine and labetalol were recorded. The postoperative pain, as assessed on the VAS, postoperative nausea and vomiting, and administration of rescue medications were also recorded. Occurrence of other adverse events, such as upper airway obstruction, respiratory depression, emergence agitation, and postoperative awareness, were recorded.

The sample size was calculated based on the previous finding that the VAS mean of surgical field was $3 \pm 1.2$ under propofolremifentanil [9] and that a difference in incidence of at least 30\% would be clinically relevant. Thus, for $\alpha=0.05,18$ patients per group were required. Assuming a loss of $10 \%$ in the follow-up period, a total sample size of 40 was needed to achieve a power of $80 \%$.

The statistical analyses were performed with SPSS 14.0 (SPSS Inc., Chicago, IL, USA). The continuous and categorical variables were analyzed using a t-test and Chi-square test, respectively. The MBP and HR were repeatedly measured, and were analyzed using a repeated measured one-way ANOVA. The nonparametric data were evaluated with an $\mathrm{X}^{2}$-test or Fisher's exact test, as appropriate. A P value under 0.05 was considered significant.

\section{Results}

Of the forty-three patients initially enrolled in the study, the data from thirty-nine patients were successfully analyzed. Four patients were dropped from the study for unrecognized upper respiratory infection, unrecognized arrhythmia, and conversion to open surgery. The patients' characteristics did not differ between the two groups (Table 1).
The median (interquartile range) of NRS for the visualization satisfaction was 7 (3) in the dexmedetomidine group and 7 (2) in the remifentanil group, and the difference between the two groups was not significantly different $(\mathrm{P}=0.95)$. The satisfaction scores for the visualization of the surgical field in each patient group are shown in Fig. 1.

There were no differences between the groups in terms of MBP (Fig. 2) or HR (Fig. 3). Eight patients in the dexmedetomidine group received labetalol just once and one patient received labetalol twice. The consumption of propofol did not differ between the two groups. In the remifentanil group, the mean consumption of remifentanil was $775.2 \pm 354.9 \mu \mathrm{g}$ and in the dexmedetomidine group, the mean consumption of dexmedetomidine was $58.3 \pm 23.4 \mu \mathrm{g}$.

The recovery profiles are presented in Table 2. The extubation time was significantly shorter in the dexmedetomidine group $(8.4 \pm 1.8 \mathrm{~min})$ than in the remifentanil group $(11.9 \pm 5.4 \mathrm{~min})$ $(\mathrm{P}=0.04)$. Except for the extubation time, the recovery profiles of the two groups were comparable at all observation periods. No adverse event occurred in either group.

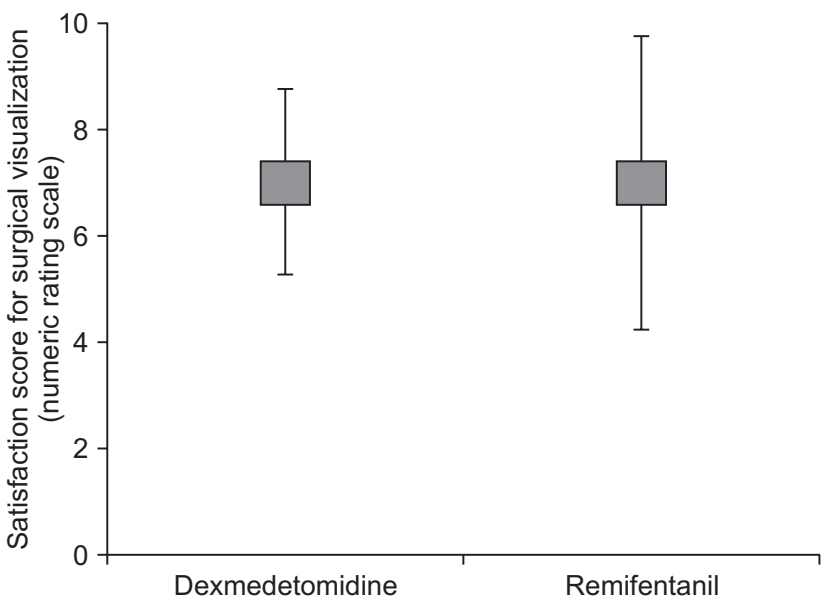

Fig. 1. Satisfaction scores with visualization of the surgical field in each patient group. The difference between the two groups was not significant.

Table 1. Demographic and Clinical Characteristics of Patients

\begin{tabular}{lccc}
\hline & Dexmedetomidine group $(\mathrm{n}=18)$ & Remifentanil group $(\mathrm{n}=21)$ & P value \\
\hline Age $(\mathrm{yr})$ & $39.2 \pm 12.5$ & $36.2 \pm 15.1$ & 0.49 \\
Gender $(\mathrm{M} / \mathrm{F})$ & $14 / 7$ & $9 / 9$ & 0.32 \\
ASA classification (I/II) & $16 / 2$ & $18 / 3$ & 0.55 \\
Height $(\mathrm{cm})$ & $168.6 \pm 7.7$ & $163.9 \pm 14.3$ & 0.24 \\
Weight $(\mathrm{kg})$ & $66.7 \pm 10.7$ & $66.3 \pm 12.6$ & 0.91 \\
Preoperative CT grade & $7.0 \pm 5.4$ & $7.4 \pm 5.5$ & 0.22 \\
Operation duration $(\mathrm{min})$ & $50.9 \pm 26.7$ & $64.8 \pm 30.7$ & 0.15 \\
Anesthesia duration $(\mathrm{min})$ & $83.1 \pm 27.7$ & $96.1 \pm 31.3$ & 0.19 \\
Propofol consumption $(\mu \mathrm{g} / \mathrm{kg} / \mathrm{min})$ & $122.1 \pm 26.8$ & $111.8 \pm 19.9$ & 0.23 \\
\hline
\end{tabular}

The values are presented as numbers or means \pm SD. F: female, M: male, ASA: American Society of Anesthesiologists, CT: computed tomography. 


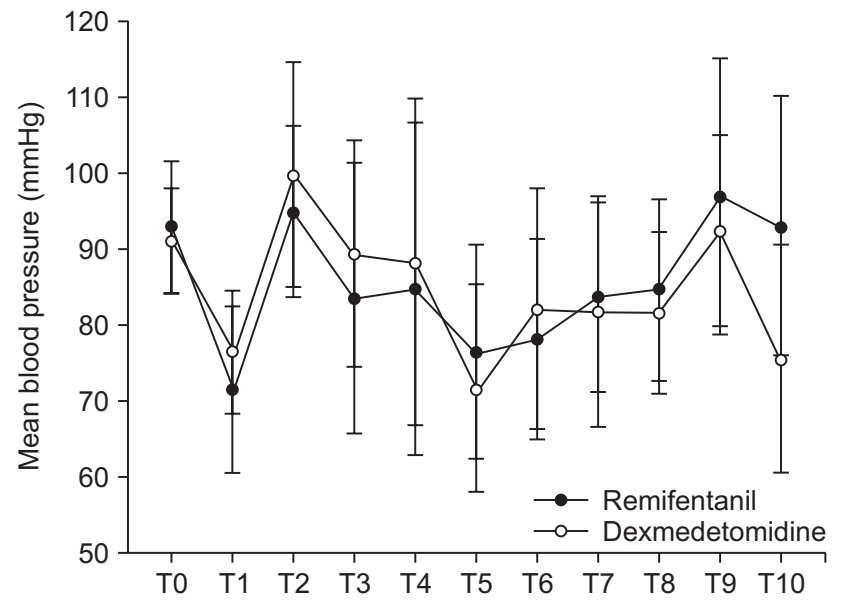

Fig. 2. Comparison of changes in mean blood pressure between dexmedetomidine group and remifentanil group. T0: before induction, T1: before intubation, T2: after intubation, T3: after local injection, T4: $5 \mathrm{~min}$ after local injection, T5: $10 \mathrm{~min}$ after local injection, T6: 20 min after local injection, T7: 5 min before end of operation, T8: end of operation, T9: extubation, T10: admission to the PACU.

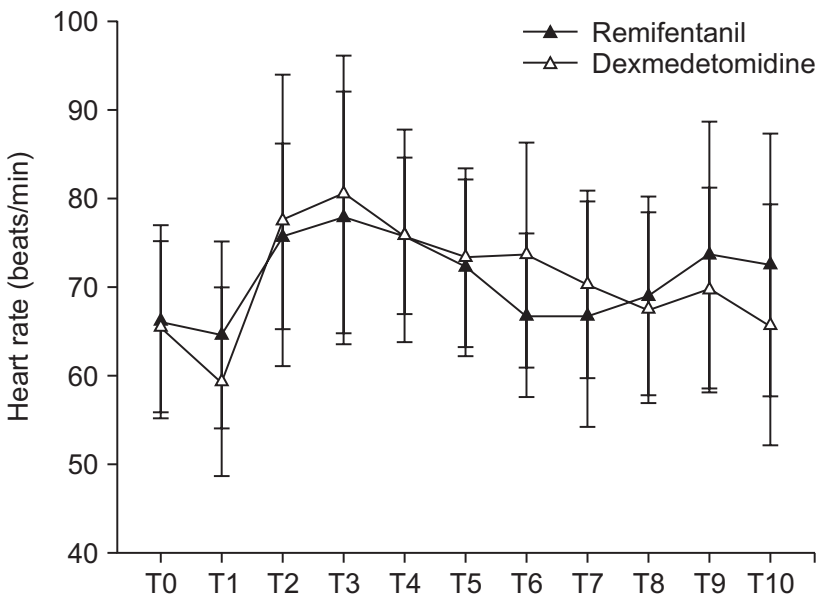

Fig. 3. Comparison of changes in heart rate between dexmedetomidine group and remifentanil group. T0: before induction, T1: before intubation, T2: after intubation, T3: after local injection, T4: 5 min after local injection, T5: $10 \mathrm{~min}$ after local injection, T6: $20 \mathrm{~min}$ after local injection, T7: 5 min before end of operation, T8: end of operation, T9: extubation, T10: admission to the PACU.

Table 2. Recovery Profiles of Patients

\begin{tabular}{lccc}
\hline & Dexmedetomidine group $(\mathrm{n}=18)$ & Remifentanil group $(\mathrm{n}=21)$ & P value \\
\hline Awake time (min) & $7.1 \pm 2.4$ & $9.4 \pm 5.3$ & 0.17 \\
Extubation time (min) & $8.4 \pm 1.8$ & $11.9 \pm 5.4$ & 0.04 \\
Respiratory rate in PACU (breaths/min) & $14.7 \pm 4.3$ & $14.5 \pm 4.3$ & 0.85 \\
Sedation scale in PACU & $2(1)$ & $2(1)$ & 0.84 \\
Postoperative pain score (VAS) & $2(2)$ & $3(2)$ & 0.14 \\
Incidence of PONV (\%) & $2(11.1)$ & $2(9.5)$ & 0.87
\end{tabular}

The values are presented as means \pm SD or numbers (IQR or \%). Awake time: from discontinuation of propofol to eye opening in response to verbal command, Extubation time: from discontinuation of propfol to extubation, PACU: postanesthetic care unit, IQR: interquartile range, VAS: visual analog score, PONV: postoperative nausea vomiting.

\section{Discussion}

The main finding of this prospective double-blind randomized study was that intraoperative continuous dexmedetomidine infusions provide similar visualization of the surgical field and similar hemodynamic changes as remifentanil TCIs during ESS. In addition, the extubation time was shorter in the dexmedetomidine group, and other recovery profiles were statistically comparable.

As the visualization of the surgical field is directly connected to the postoperative outcomes, the reduction of surgical bleeding has been a major concern of otolaryngologists and anesthesiologists [2-4]. In previous studies, the visualization of the surgical field was founded to be directly correlated with the HR $[10,11]$. In particular, remifentanil was found to provide better visualization of the surgical field, suggesting that this shortacting opioid agent could reduce the HR and MBP immediately and effectively [12]. Thus, the continuous infusion of remifent- anil has become a reliable strategy for the superior visualization of the surgical field in ESS [5].

Similarly to remifentanil, dexmedetomidine has been shown to have positive effects on the visualization of the surgical field in several ESS-related studies $[6,13,14]$. This improvement may be caused by the peripheral vasoconstrictive effect of the $\alpha_{2}$ agonist. However, in existing ESS-related studies, the patients in the placebo group had a significantly higher HR than those in the dexmedetomidine group. It is preferable for the visualization of the surgical field to be achieved through reduction of the HR rather than through vasoconstriction. The present study set limits for HR and MBP, and demonstrated similar hemodynamic changes in the two groups.

To minimize the degree to which the severity and extension of the disease affect the bleeding, we estimated the preoperative Lund-Mackay CT score of the patients. The radiologic scoring system is part of the Lund-Mackay staging system and is used more widely than symptom scores and endoscopic scores. As the 
interpretation of CT scans does not require radiology training and is unambiguous, results can be expressed as simple numeric scores. As a result, this method has been shown to present high interobserver reliability [15]. The patients with higher LundMackay preoperative scores presented more risks of complications [7]. The scores were not significantly different between the two groups in this study. As there was only a small number of patients with high Lund-Mackey preoperative scores in our results, it is difficult to clarify the effect of this score on the visualization of the ESS surgical field. Further study is needed to better understand the contribution of this score to the visualization of the surgical field.

The vasoconstrictive effect of intravenous dexmedetomidine has been widely demonstrated in animal models [16,17]. Moreover, experimental research has provide evidence that intravenous dexmedetomidine has a vasoconstrictive effect on the human arterial and venous system $[18,19]$. However, there is a lack of direct evidence for the fact that intravenous dexmedetomidine contracts the peripheral vessels directly and reduces surgical bleeding during actual operations. Intravenous dexmedetomidine appears to reduce surgical bleeding and to improve the visualization of the surgical field in ESS only.

Although dexmedetomidine has an analgesic effect, its analgesic property is weaker than that of remifentanil [20]. In the present study, the patients in the dexmedetomidine group were administered fentanyl and dexmedetomidine as analgesic agents. It is uncertain whether $1 \mu \mathrm{g} / \mathrm{kg}$ of fentanyl can provide sufficient analgesia in the induction period. If tachycardia or hypertension occurred afterwards, labetalol was administered to maintain the $\mathrm{HR}$ and MBP within $20 \%$ of the preoperative resting values. It is unclear whether this administration of labelatol could have influenced the local vasoconstriction in the nasal mucosa. However, the labetalol administration could not have had any signifi- cant effect on the present result, as the vascularity of the nasal polypoid tissue is sparse [21], and labetalol has a weak blocking potency for the $\alpha 1$ - adrenergic receptor. [22]

In the present study, the continuous infusion of dexmedetomidine presented similar recovery profiles to remifentanil TCIs. There have been several reports of recovery profiles after the administration of intravenous dexmedetomidine during general anesthesia. Most studies demonstrated that the perioperative use of dexmedetomidine can reduce the postoperative opioid consumption, pain intensity, and need for antiemetic therapy $[23,24]$. Furthermore, there has been report that the use of intravenous dexmedetomidine in ESS caused less emergence agitation and improved the quality of recovery [25]. In this study, we discontinued the dexmedetomidine five minutes before the end of the surgery, and the remifentanil directly after extubation. The comparison of the recovery profiles between the two agents is less precise due to the difference in discontinuation times. Those results supporting the favorable recovery effects of dexmedetomidine, which are comparable to those of remifentanil, indicate that the perioperative use of dexmedetomidine is a good option to guarantee the smooth emergence of high risk patients.

The limitations of this study should be mentioned. First, while the patients who received remifentanil TCIs served as a positive control group, the study did not include a placebo group, which would have provide a useful contrast with the dexmedetomidine effect. Second, it was hard to titrate the remifentanil and dexmedetomidine to equipotent analgesic doses, as two agents have different pharmacologic properties.

In conclusion, the present study showed that continuous dexmedetomidine infusions provide a similar visualization of the surgical field to remifentanil TCIs in patients undergoing ESS. Furthermore, dexmedetomidine allows for comparably hemodynamic stability and faster recovery than remifentanil TCI.

\section{References}

1. Stammberger H, Posawetz W. Functional endoscopic sinus surgery. Concept, indications and results of the Messerklinger technique. Eur Arch Otorhinolaryngol 1990; 247: 63-76.

2. Stankiewicz JA. Complications of endoscopic intranasal ethmoidectomy. Laryngoscope 1987; 97: 1270-3.

3. Stankiewicz JA. Complications of endoscopic sinus surgery. Laryngoscope 1989; 99: 686-90.

4. Maniglia AJ. Fatal and other major complications of endoscopic sinus surgery. Laryngoscope 1991; 101: 349-54.

5. Rodriguez Valiente A, Roldan Fidalgo A, Laguna Ortega D. Bleeding control in endoscopic sinus surgery: a systematic review of the literature. Rhinology 2013; 51: 298-305.

6. Guven DG, Demiraran Y, Sezen G, Kepek O, Iskender A. Evaluation of outcomes in patients given dexmedetomidine in functional endoscopic sinus surgery. Ann Otol Rhinol Laryngol 2011; 120: 586-92.

7. Hopkins C, Browne JP, Slack R, Lund V, Brown P. The Lund-Mackay staging system for chronic rhinosinusitis: how is it used and what does it predict? Otolaryngol Head Neck Surg 2007; 137: 555-61.

8. Alon E, Baitella L, Hossli G. Double-blind study of the reversal of midazolam-supplemented general anaesthesia with Ro 15-1788. Br J Anaesth 1987; 59: 455-8.

9. Ragab SM, Hassanin MZ. Optimizing the surgical field in pediatric functional endoscopic sinus surgery: a new evidence-based approach. 
Otolaryngol Head Neck Surg 2010; 142: 48-54.

10. Eberhart LH, Folz BJ, Wulf H, Geldner G. Intravenous anesthesia provides optimal surgical conditions during microscopic and endoscopic sinus surgery. Laryngoscope 2003; 113: 1369-73.

11. Nair S, Collins M, Hung P, Rees G, Close D, Wormald PJ. The effect of beta-blocker premedication on the surgical field during endoscopic sinus surgery. Laryngoscope 2004; 114: 1042-6.

12. Ahn HJ, Chung SK, Dhong HJ, Kim HY, Ahn JH, Lee SM, et al. Comparison of surgical conditions during propofol or sevoflurane anaesthesia for endoscopic sinus surgery. Br J Anaesth 2008; 100: 50-4.

13. Goksu S, Arik H, Demiryurek S, Mumbuc S, Oner U, Demiryurek AT. Effects of dexmedetomidine infusion in patients undergoing functional endoscopic sinus surgery under local anaesthesia. Eur J Anaesthesiol 2008; 25: 22-8.

14. Dogan R, Erbek S, Gonencer HH, Erbek HS, Isbilen C, Arslan G. Comparison of local anaesthesia with dexmedetomidine sedation and general anaesthesia during septoplasty. Eur J Anaesthesiol 2010; 27: 960-4.

15. Oluwole M, Russell N, Tan L, Gardiner Q, White P. A comparison of computerized tomographic staging systems in chronic sinusitis. Clin Otolaryngol Allied Sci 1996; 21: 91-5.

16. Flacke JW, Flacke WE, Bloor BC, McIntee DF. Hemodynamic effects of dexmedetomidine, an alpha 2-adrenergic agonist, in autonomically denervated dogs. J Cardiovasc Pharmacol 1990; 16: 616-23.

17. Lawrence CJ, Prinzen FW, de Lange S. Hemodynamic and coronary vascular effects of dexmedetomidine in the anesthetized goat. Acta Anaesthesiol Scand 1997; 41: 830-6.

18. Talke P, Lobo E, Brown R. Systemically administered alpha2-agonist-induced peripheral vasoconstriction in humans. Anesthesiology 2003; 99: 65-70.

19. Muszkat M, Kurnik D, Sofowora GG, Wood AJ, Stein CM. Independent regulation of $\alpha 1$ and $\alpha 2$ adrenergic receptor-mediated vasoconstriction in vivo. J Hypertens 2011; 29: 251-6.

20. Cortinez LI, Hsu YW, Sum-Ping ST, Young C, Keifer JC, Macleod D, et al. Dexmedetomidine pharmacodynamics: Part II: Crossover comparison of the analgesic effect of dexmedetomidine and remifentanil in healthy volunteers. Anesthesiology 2004; 101: 1077-83.

21. Mygind N, Dahl R, Bachert C. Nasal polyposis, eosinophil dominated inflammation, and allergy. Thorax 2000; 55 Suppl 2: 680-3.

22. Richards DA, Prichard BC, Boakes AJ, Tuckman J, Knight EJ. Pharmacological basis for antihypertensive effects of intravenous labetalol. Br Heart J 1977; 39: 99-106

23. Tufanogullari B, White PF, Peixoto MP, Kianpour D, Lacour T, Griffin J, et al. Dexmedetomidine infusion during laparoscopic bariatric surgery: the effect on recovery outcome variables. Anesth Analg 2008; 106: 1741-8.

24. Ohtani N, Yasui Y, Watanabe D, Kitamura M, Shoji K, Masaki E. Perioperative infusion of dexmedetomidine at a high dose reduces postoperative analgesic requirements: a randomized control trial. J Anesth 2011; 25: 872-8.

25. Kim SY, Kim JM, Lee JH, Song BM, Koo BN. Efficacy of intraoperative dexmedetomidine infusion on emergence agitation and quality of recovery after nasal surgery. Br J Anaesth 2013; 111: 222-8. 